\title{
WEIGHTED NORM INEQUALITIES FOR THE HARDY-LITTLEWOOD MAXIMAL FUNCTION
}

\author{
WO-SANG YOUNG
}

\begin{abstract}
A characterization is obtained for weight functions $v$ for which the Hardy-Littlewood maximal operator is bounded from $I^{\prime \prime}\left(\mathbf{R}^{\prime \prime}, w d x\right)$ to $I^{\prime \prime}\left(\mathbf{R}^{\prime \prime}, v d x\right)$ for some nontrivial $n$ :
\end{abstract}

In this note we obtain a necessary and sufficient condition on weight functions $v \geqslant 0$ such that the Hardy-Littlewood maximal operator is bounded from $L^{p}\left(\mathbf{R}^{n}, w d x\right)$ to $L^{p}\left(\mathbf{R}^{n}, v d x\right)$ for some $w<\infty$ a.e. This answers a question posed by B. Muckenhoupt in [3]. The problem of characterizing all weight functions $w \geqslant 0$ for which there are nontrivial $v$ 's was solved independently by J. L. Rubio de Francia [4] and L. Carleson and P. W. Jones [1].

Let $M$ be the Hardy-Littlewood maximal operator defined by

$$
M f(x)=\sup _{r>0} \frac{1}{|B(x, r)|} \int_{B(x, r)}|f| d y,
$$

where $B(x, r)$ is the ball of radius $r$ centered at $x$ and $|B(x, r)|$ is its Lebesgue measure. Our result is as follows.

THEOREM. Given $v \geqslant 0$ and $1<p<\infty$, the following conditions are equivalent:

(a) There is $w<\infty$ a.e. such that

for all $f \in L^{p}\left(\mathbf{R}^{n}, w d x\right)$.

$$
\int_{\mathbf{R}^{n}}|M f|^{p} v d x \leqslant C \int_{\mathbf{R}^{n}}|f|^{p} w d x
$$

(b)

$$
\int_{\mathbf{R}^{n}} \frac{v(x)}{\left(1+|x|^{n}\right)^{p}} d x<\infty .
$$

In this paper $C$ denotes a constant depending only on $n$ and $p$, and may vary from line to line.

In [4] Rubio de Francia observed that (a) implies

$$
u \in L_{\mathrm{loc}}^{1}\left(\mathbf{R}^{n}\right) \text { and }\left(\int_{|x| \leqslant R} u\right)^{1 / p}=O\left(R^{n}\right) \quad(R \rightarrow \infty)
$$

Received by the editors September 1, 1981.

1980 Mathematics Subject Classification. Primary 42B25.

${ }^{1}$ Research supported in part by Natural Sciences and Engineering Research Council Canada Grant No. A5165.

(c) 1982 American Mathematical Society 0002-9939/81/0000-1109/\$01.75 
and conjectured that it is also sufficient for (a). This is not the case since $u(x)=\mid x$ $\left.\right|^{n(p-1)}$ satisfies (*) but not (b).

Proof of Theorem. We first show that (a) implies (b). Since $w \neq \infty$, there is a set $A$ with positive measure in which $w$ is bounded. Moreover, we can assume $A \subset\{\mid x$ $\mid \leqslant R\}$ for some $1<R<\infty$. Let $f=\chi_{A}$. Then

$$
\int_{\mathbf{R}^{n}}|f|^{p} w d x=\int_{A} w<\infty
$$

For $|x| \leqslant R, M f(x) \geqslant C|A| / R^{n}$, and for $|x|>R, M f(x) \geqslant C|A| /|x|^{n}$. Hence

$$
\int_{\mathbf{R}^{n}}|M f|^{p} v d x \geqslant\left(C \frac{|A|}{R^{n}}\right)^{p} \int_{\mathbf{R}^{n}} \frac{v(x)}{\left(1+|x|^{n}\right)^{p}} d x .
$$

Therefore

$$
\int_{\mathbf{R}^{n}} \frac{v(x)}{\left(1+|x|^{n}\right)^{p}} d x<\infty
$$

We now prove that (b) implies (a). Let $v_{1}(x)=v(x)$ if $v(x) \geqslant 1$, and $v_{1}(x)=1$ if $v(x)<1$. Then $v_{1}$ also satisfies (b) because

$$
\int_{\mathbf{R}^{n}} \frac{v_{1}(x)}{\left(1+|x|^{n}\right)^{p}} d x \leqslant \int_{\mathbf{R}^{n}} \frac{v(x)+1}{\left(1+|x|^{n}\right)^{p}} d x<\infty .
$$

Since $v \leqslant v_{1}$, (a) is satisfied if we show that there is $w<\infty$ a.e. such that

$$
\int_{\mathbf{R}^{n}}|M f|^{p} v_{1} d x \leqslant C \int_{\mathbf{R}^{n}}|f|^{p} w d x
$$

for all $f \in L^{p}\left(\mathbf{R}^{n}, w d x\right)$.

Let $u(x)=\left(1+|x|^{n}\right)^{1-p}$. We observe that $M\left(u v_{1}\right)<\infty$ a.e. To see this let $1<R<\infty$ and consider $\{|x| \leqslant R\}$. For any such $x$,

$$
\sup _{r \leqslant R} \frac{1}{r^{n}} \int_{|y-x| \leqslant r} u v_{1} \leqslant C M\left(v_{1} \chi_{\{|y| \leqslant 2 R\}}\right)(x) .
$$

$M\left(v_{1} \chi_{\{|y| \leqslant 2 R\}}\right)$ is finite a.e. because $v_{1} \in L_{\text {loc }}^{1}\left(\mathbf{R}^{n}\right)$. Also, for $r>R$

$$
\frac{1}{r^{n}} \int_{|y-x| \leqslant r} u v_{1} \leqslant \frac{1}{r^{n}} \int_{|y| \leqslant 2 r} \frac{v_{1}(y)}{\left(1+|y|^{n}\right)^{p-1}} d y \leqslant C \int_{\mathbf{R}^{n}} \frac{v_{1}(y)}{\left(1+|y|^{n}\right)^{p}} d y<\infty .
$$

Hence $M\left(u v_{1}\right)<\infty$ a.e.

Let $w=u^{-3} M\left(u v_{1}\right)$. Then $w<\infty$ a.e. We shall show that (1) holds for this $w$. Let $f \in L^{p}\left(\mathbf{R}^{n}, w d x\right)$, and, for $k=0,1,2, \ldots$, let $f_{k}=f \chi_{\left\{2^{k} \leqslant|x|<2^{k+1}\right\}}$. Then it follows from Fefferman and Stein [2] that

$$
\begin{aligned}
\int_{|x| \leqslant 2^{k+2}}\left|M f_{k}\right|^{p} v_{1} & \leqslant C 2^{k n(p-1)} \int_{\mathbf{R}^{n}}\left|M f_{k}\right|^{p} u v_{1} \\
& \leqslant C 2^{k n(p-1)} \int_{\mathbf{R}^{n}}\left|f_{k}\right|^{p} M\left(u v_{1}\right) \\
& \leqslant C 2^{-2 k n(p-1)} \int_{\mathbf{R}^{n}}|f|^{p} w
\end{aligned}
$$


For $|x|>2^{k+2}$, we have

$$
\begin{aligned}
M f_{k}(x) & \leqslant \frac{C}{|x|^{n}} \int_{2^{k} \leqslant|x|<2^{k+1}}|f| \\
& \leqslant \frac{C}{|x|^{n}}\left(\int_{\mathbf{R}^{n}}|f|^{p} w\right)^{1 / p}\left(\int_{2^{k} \leqslant|x|<2^{k+1}} w^{-1 /(p-1)}\right)^{1-1 / p},
\end{aligned}
$$

by Hölder's inequality. Therefore

$$
\int_{|x|>2^{k+2}}\left|M f_{k}\right|^{p} v_{1} \leqslant C\left(\int_{\mathbf{R}^{n}} \frac{v_{1}(x)}{\left(1+|x|^{n}\right)^{p}} d x\right)\left(\int_{\mathbf{R}^{n}}|f|^{p} w\right)\left(\int_{2^{k} \leqslant|x|<2^{k+1}} w^{-1 /(p-1)}\right)^{p-1} .
$$

To estimate the last factor we note that for $2^{k} \leqslant|x|<2^{k+1}$,

$$
M\left(u v_{1}\right)(x) \geqslant \frac{C}{2^{k n}} \int_{|y| \leqslant 2^{k}} \frac{v_{1}(y)}{\left(1+|y|^{n}\right)^{p-1}} d y \geqslant C 2^{-k n(p-1)},
$$

since $v_{1} \geqslant 1$. Hence, $w(x) \geqslant C 2^{2 k n(p-1)}$, and

$$
\left(\int_{2^{k} \leqslant|x|<2^{k+1}} w^{-1 /(p-1)}\right)^{p-1} \leqslant 2^{-k n(p-1)} .
$$

Altogether we have

$$
\int_{\mathbf{R}^{n}}\left|M f_{k}\right|^{p} v_{1} \leqslant C\left[\int_{\mathbf{R}^{n}} \frac{v(x)}{\left(1+|x|^{n}\right)^{p}} d x+1\right]\left(\int_{\mathbf{R}^{n}}|f|^{p} w\right) 2^{-k n(p-1)} .
$$

It then follows from Minkowski's inequality that

$$
\begin{aligned}
\int_{\mathbf{R}^{n}}|M f|^{p} v_{1} & \leqslant\left[\sum_{k=0}^{\infty}\left(\int_{\mathbf{R}^{n}}\left|M f_{k}\right|^{p} v_{1}\right)^{1 / p}\right]^{p} \\
& \leqslant C\left[\int_{\mathbf{R}^{n}} \frac{v(x)}{\left(1+|x|^{n}\right)^{p}} d x+1\right]\left(\int_{\mathbf{R}^{n}}|f|^{p} w\right)\left[\sum_{k=0}^{\infty} 2^{-k n(1-1 / p)}\right]^{p} \\
& \leqslant C\left[\int_{\mathbf{R}^{n}} \frac{v(x)}{\left(1+|x|^{n}\right)^{p}} d x+1\right]\left(\int_{\mathbf{R}^{n}}|f|^{p} w\right)
\end{aligned}
$$

This complete the proof of the theorem.

I would like to thank R. A. Hunt, D. S. Kurtz and B. Muckenhoupt for their helpful conversations.

\section{REFERENCES}

1. L. Carleson and P. W. Jones, Weighted norm inequalities and a theorem of Koosis, Mittag-Leffler Rep. No. 2, 1981 .

2. C. Fefferman and E. M. Stein, Some maximal inequalities, Amer. J. Math. 93 (1971), 107-115.

3. B. Muckenhoupt, Weighted norm inequalities for classical operators, Proc. Sympos. Pure Math., vol. 35, part 1, Amer. Math. Soc., Providence, R. I., 1979, pp. 69-83.

4. J. L. Rubio de Francia, Boundedness of maximal functions and singular integrals in weighted $L^{p}$ spaces, Proc. Amer. Math. Soc. 83 (1981), 673-679.

Department of Mathematics, University of Alberta, Edmonton, Alberta T6G 2Gl, Canada 